\title{
Anesthetic management for emergent Cesarean section in a patient with toxic epidermal necrolysis -A case report-
}

\author{
Jung Hyang Lee ${ }^{1}$, Hyeon Jeong Yang ${ }^{1}$, Byeong-Kuk Yang ${ }^{1}$, Su-Yeon Lee ${ }^{1}$, Chunghyun Park ${ }^{1}$, and \\ Dong-Hyun Kim $^{2}$ \\ Departments of ${ }^{1}$ Anesthesiology and Pain Medicine, ${ }^{2}$ Dermatology, College of Medicine, CHA University, Seongnam, Korea
}

Toxic epidermal necrolysis (TEN) is rare but serious cutaneous reaction with significant mortality and long-term morbidity. Various etiologies, particularly numerous medications and infectious agents have been implicated. It is characterized as inflammatory bullous lesions of the skin and mucous membrane and can develop serious complications such as pneumonia, pneumothorax, sepsis and renal failure. In general, patients with TEN are managed as severe second-degree burn patients with preventing excessive fluid deficit and infections. In this case, we aimed to present anesthetic management of a 26 -year-old pregnant woman with TEN who received general anesthesia during emergent cesarean section. (Korean J Anesthesiol 2010; 59: S167-S171)

Key Words: Cesarean section, General anesthesia, Toxic epidermal necrolysis.

Toxic epidermal necrolysis (TEN), which shares the same spectrum as the Stevens-Johnson syndrome (SJS), is an emergent cutaneous disease accompanying serious systemic complications, along with erythema, vesicle and erosion of the skin and mucous membranes [1]. Patients are diagnosed with SJS if less than $10 \%$ of their whole-body skin is infiltrated, while TEN needs $30 \%$ or more. If the range is between $10 \%$ and $30 \%$, the patients are categorized as an overlap of SJS and TEN [2]. One of the typical etiologies is use of drugs such as sulfonamides, carbamazepine, NSAIDs (oxicam), allopurinol, chlormezanone, and corticosteroids. Infection, radiation therapy, and malignant neoplasm also can inflict serious cutaneous diseases [3]. In addition to skin lesions, SJS and TEN can infiltrate the mucous membranes of some organs, including eye, mouth, airway, esophagus, rectum, and vaginal canal and then cause erosions and ulcers. Both illnesses can also lead to more serious complications, for example, pneumothorax, pneumonia, hepatic lesion, sepsis, and renal failure [4-6].

This case involves a pregnant woman who contracted TEN after she started taking tocolytics on the 2nd day of the 28th week of pregnancy. When being treated for TEN, she didn't show any complications except for lesions of the skin and mucous membranes. However, her lesions worsened abruptly and premature labor continued, resulting in an emergency Cesarean section. For its uniqueness, we report the case herein with reference to the related literature.

Received: June 1, 2010. Revised: 1st, June 17, 2010; 2nd, July 8, 2010. Accepted: July 19, 2010.

Corresponding author: Hyeon Jeong Yang, M.D., Department of Anesthesiology and Pain Medicine, College of Medicine, CHA University, 351, Yatap-dong, Bundang-gu, Seongnam 463-828, Korea. Tel: 82-31-780-1838, Fax: 82-31-701-9433, E-mail: yanghj@medigate.net

(c) This is an open-access article distributed under the terms of the Creative Commons Attribution Non-Commercial License (http:// creativecommons.org/licenses/by-nc/3.0/), which permits unrestricted non-commercial use, distribution, and reproduction in any medium, provided the original work is properly cited. 


\section{Case Report}

The patient was a 26-year-old and body weight of $61 \mathrm{~kg}$ pregnant woman, who was transferred from the previous hospital where the treatment for her urticaria and premature labor was unsuccessful. She entered our hospital on the 3rd day of the 31st week of pregnancy. She had been on ritodrine and $\mathrm{MgSO}_{4}$ for the treatment of premature labor since the 2nd day of the 28th week of pregnancy. The patient also suffered from erythema and pruritus spreading through her entire body, including the face. After receiving her, we began to administrate a local steroid with the suspicion of urticarial vasculitis and pemphigoid gestationis. However, her erythema and vesicles deteriorated (Fig. 1), skin started to peel, and oral mucous edema and ulcers worsened, prompting us to conduct a punch biopsy. Since the test found a subepidermal blister with confluent keratinocyte necrosis, we diagnosed the patient with TEN and initiated more intensive care such as administration of immunoglobulin and oral steroids. However, her skin and mucous membranes continued to deteriorate rapidly, and her problems with premature labor remained uncontrolled. After all, we decided to stop giving her ritodrine and $\mathrm{MgSO}_{4}$ and to conduct an emergency Cesarean section. It was only the 9th day since the patient came in our hospital.

Before the operation, the parturient complained of a sore throat and had severe edema on her mucous membrane and tongue, accompanied by erythema and ulcers. The results of blood and biochemical tests were Hb $10.3 \mathrm{~g} / \mathrm{dl}$, Hct 29.7\%, WBC $5.74 \times 10^{9} / \mathrm{L}$, BUN $3.0 \mathrm{mg} / \mathrm{dl}, \mathrm{Cr} 0.6 \mathrm{mg} / \mathrm{dl}$, AST $25 \mathrm{IU} / \mathrm{L}, \mathrm{Na} 141$ $\mathrm{mEq} / \mathrm{L}$, and $\mathrm{K} 3.5 \mathrm{mEq} / \mathrm{L}$. There was no sign of dehydration or abnormality in the electrolytes, but ALT was $61 \mathrm{IU} / \mathrm{L}$, which is

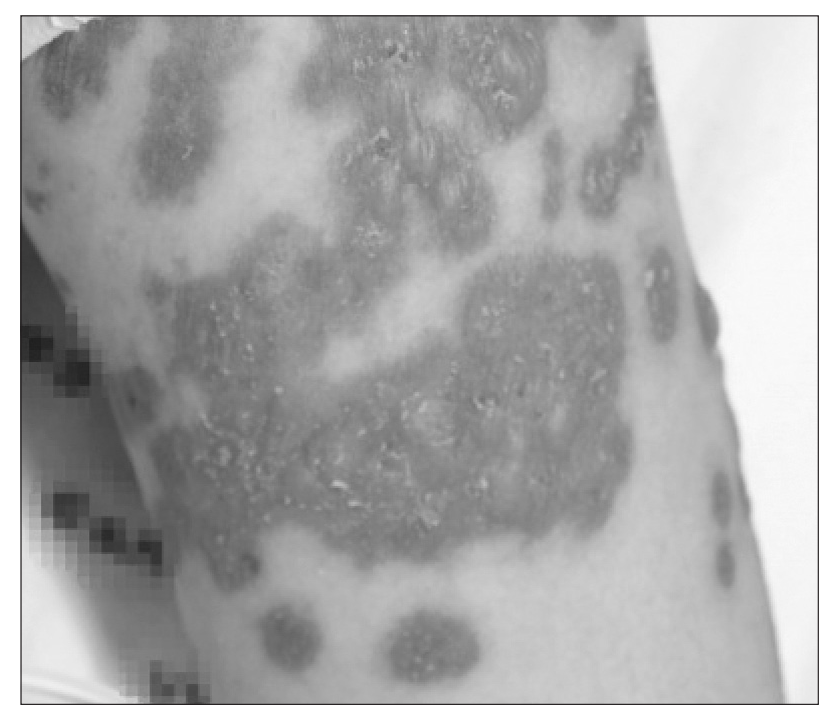

Fig. 1. This photograph shows preoperative skin change of right arm. a little bit higher than a normal level. Her chest radiograph and electrocardiogram also didn't indicate any abnormality. Her vital signs were stable when she arrived in the operating room: blood pressure was 130/60 mmHg; pulse was $80 \mathrm{bpm}$; oxygen saturation was $100 \%$; and temperature was $37^{\circ} \mathrm{C}$. Initially, we planned to apply regional anesthesia since her oral edema seemed to make it difficult to secure the airway and dangerous to insert and remove a endotracheal tube. However, we changed our mind to general anesthesia since the skin lesions expanded to the whole area of her back and waist, which meant that there was high chance of infection for the regional anesthetic areas.

We secured one of her peripheral veins on her right leg by using a 16-guage catheter to supply sufficient fluid, even though there was no sign of dehydration. Also, we inserted a 20-guage catheter into her left radial artery for accurate blood pressure measurement and blood sampling. When conducting the endotracheal intubation, we used gauze instead of bite block in order to fix the tube due to her serious skin damage. To induce prompt anesthesia, $120 \mathrm{mg}$ of propofol and $70 \mathrm{mg}$ of succinylcholine were administered. To minimize the damage on the larynx and bronchus, we put vaseline gel at the end of the laryngoscope, spread lidocaine on the cuff of the endotracheal tube, and used a small tube, whose internal diameter was just $6.5 \mathrm{~mm}$, with only one strip of paper tape to fix the tube. The tube was inserted smoothly without any resistance and did not cause a burst of blebs and bleeding inside the mouth. We did not insert a bite block to prevent further intraoral damage.

To reduce a barotrauma, we decided $8 \mathrm{ml}$ as a tidal volume per $1 \mathrm{~kg}$ of body weight, thus providing $460 \mathrm{ml}$ total. We maintained 14 per 1 minute as the respiration rate and $30-32 \mathrm{mmHg}$ for $\mathrm{PaCO}_{2}$. Two liters of air and two liters of $\mathrm{O}_{2}$ were provided every one minute and, to maintain anesthesia while decreasing liver damage, $0.8-1.0$ vol\% of isoflurane was supplied. Also, we kept her body temperature at $36.4-36.8^{\circ} \mathrm{C}$ while measuring the temperature through her ear every 5 minute. We maintained the inspiratory pressure at $15-16 \mathrm{cmH}_{2} \mathrm{O}$ throughout the operation and, to minimize the maximum level of the inspiratory pressure, which was elevated by an increase in the abdominal pressure, we used manual ventilation during the expulsion of the baby. The mother delivered an 1,885-gram son only 6 minutes after the beginning of the Cesarean section and didn't show any serious hemorrhage or unstable vital signs. We provided $100 \mu \mathrm{g}$ of fentanyl, $2.5 \mathrm{mg}$ of midazolam, and 20 iu of oxytocin while giving $100 \mathrm{mg}$ of hydrocortisone to reduce laryngopharyngeal edema and pain. Moreover, we connected continuous infuser with $1,000 \mu \mathrm{g}$ of fentanyl and Normal saline to the catheter attached to her right leg vein for controlliing postoperative pain. Bolus dose of the infuser was $0.5 \mathrm{ml}$, lock out interval was 15 minutes, and background infusion dose was $2 \mathrm{ml} / \mathrm{hr}$. When the 40-minute operation finished, we avoided intraoral suction to 
prevent intraoral damage and pulled out the endotrachal tube as smoothly as possible while reviving the patient's spontaneous breathing and consciousness. The mother was taken to the intensive care unit (ICU) via the postanesthetic recovery unit where we closely watched for her condition. She lost $500 \mathrm{ml}$ of blood and $200 \mathrm{ml}$ of urine during the surgery while we provided $1,800 \mathrm{ml}$ of crystalloid solution. Her postoperative vital signs indicated BP 120/65 mmHg, pulse $70 \mathrm{bpm}$, pulse oximeter $100 \%$, and temperature $36.5^{\circ} \mathrm{C}$ respectively. There was nothing unusual found by auscultation with stethoscopy and the patient didn't complain of either dyspnea or airway obstruction caused by edema. Since the duration of the operation was short, she did not experience fluctuation in body temperature or shivering due to hypothermia. The results of the arterial blood gas study were $\mathrm{pH}$ 7.44, $\mathrm{PCO}_{2} 34.6 \mathrm{mmHg}, \mathrm{PO}_{2} 88.1 \mathrm{mmHg}$, and Sat $\mathrm{O}_{2} 97.1$ and, according to the chest radiograph, there was no pulmonary damage inflicted by a barotrauma. We conducted visual inspection and history taking 1 hour after the Cesarean section to learn that her edema on the intraoral mucous membranes had not worsened and her sore throat had been eased. Afterward, she showed stable vital signs except a fever $\left(37.7^{\circ} \mathrm{C}\right)$ that she suffered on the 2 nd day in the ICU, without any symptom of anemia, dehydration, or abnormality in electrolytes. We just continued to administer oral and local antibiotics, and immunoglobulin. Three days after the patient was accepted to the ICU, her skin eruption and vesicles seemed to have improved (Fig. 2), and thus she was moved to the ordinary ward. Her liver function gradually normalized: her AST / ALT were 84/60, 98/34, and 21/19 (IU/L) after 2nd, 3rd, and 4 th days, respectively, after the operation. The patient consistently got better and was eventually discharged from our hospital after staying 16 days total without any specific problem.

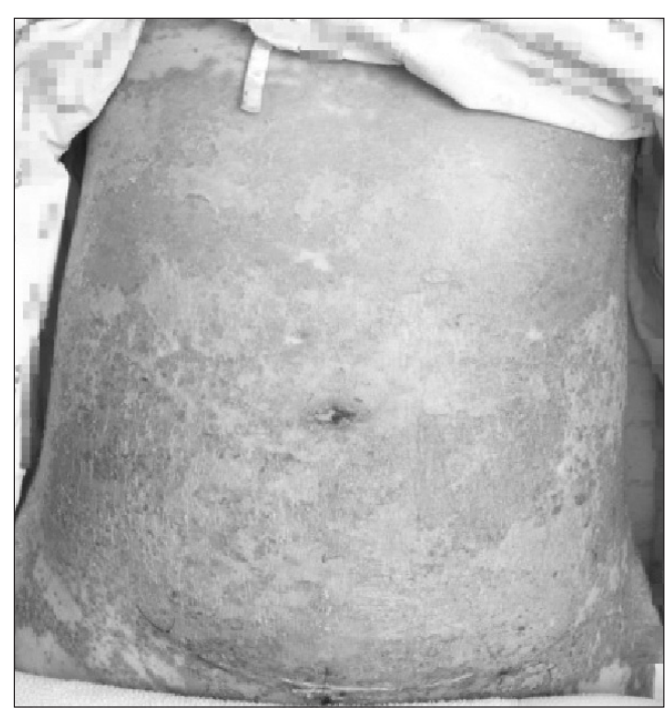

Fig. 2. This photograph shows postoperative skin change of abdomen.

\section{Discussion}

TEN, which was first introduced by Lyell in 1956, lead to the necrosis of a whole epidermis, along with a large scale of epidermolysis [1]. Although values differ depending on reports, the incidence is 1 to 3 in a million people [7] and the mortality rate is $25-30 \%$ - one of the main culprits of death is sepsis caused by secondary infection [8].

A drug is the biggest etiology and there is a report that refers to 1 patient who contracted TEN following the use of ritodrine [9]. In our case, also, ritodrine is believed to have caused the disease. As for histological symptoms, keratin-generating cells inside the epidermis are killed, resulting in both the mucosal erosion of various organs and a large scale of epidermolysis [10].

TEN begins with some prodromes such as a fever, headache, sore throat, and myalgia. Skin eruption is atypical, and, most of the time, erythema or black spot starts to appear on the face, upper body, or vicinity of limbs. Then, lesions develop into loose vesicles and ultimately show Nikolsky's sign, in which the epidermis becomes separated from the dermis. In our case as well, cutaneous lesions grew from erythema to vesicles and lastly, to epidermolysis, although sometimes the aforementioned 3 symptoms were observed simultaneously. The infiltration into mucous membranes also occurs and the mucous membrane of an eye is the most vulnerable one as seen from conjunctivitis, keratohelcosis, and uveitis. Also, it can bring about inflammation and perforation on the gastrointestinal mucous membranes, thus causing gastrointestinal hemorrhage, panperitonitis [4], hepatic lesions, and acute renal failure.

As for pulmonary complications, bronchial obstruction is reported to happen because of the erosion on the mucous membranes of the trachea and bronchiole [5]. Also, it is commonly known that bilateral pneumothorax occurs since the fistulas on the bronchus, alveolus, and pleura are formed by the rupture of the blebs in the visceral pleura [6]. Sepsis is the most severe complication, having killed the biggest number of TENcontracted patients. Sepsis is the infection of blood and body tissues, and these infections are likely to arise from skin, lungs, Foley catheters, and regions of an intravenous injection. The larger the lesions are, the more chance of infection the patient has. In our case, she suffered from erythema, vesicle, crust, and epidermolysis on her trunk and 4 limbs, as well as some part of her face. In addition, she had oral ulcers and a sore throat and the results of her liver function tests were not favorable. Nevertheless, she fortunately didn't contract serious pulmonary complications or sepsis.

There are some particular considerations regarding anesthetic management for TEN patients. First of all, doctors should have special caution for the patients' airways when they have edema, erythema, or ulcers in their mouths, throats, and airways. Also, 
providing sufficient fluid and maintaining an appropriate concentration of electrolytes are important since the patients tend to lose water and electrolytes if their whole-body skin is deprived. Pregnant patients are an object of particular attention because they are at higher risk of bleeding and trauma during tracheal intubation, even without TEN. The reasons why they are at higher risk are that their airways become narrower due to edema and mucosal capillary engorgement of airway. For those reasons, anesthesiologist should conduct endotracheal intubation as carefully as possible and should use a smaller tube (I.D., 6.0-6.5 $\mathrm{mm}$ ) for pregnant TEN patients. In our case, the mother complained of a sore throat and had intraoral edema and ulcers. Therefore, we put gel on a laryngoscope and endobronchial tube and used a small tube in order to minimize the trauma inflicted by the intubation.

Since TEN invades not only whole-body skin but also mucous membranes of various organs, the treatment for this disease is required to be more intensive than the fluid treatment for a second-degree burn, while securing large bore venous lines. In our case, the patient's lesions rapidly worsened. However, we decided not to secure her central vein because she didn't show any signs of dehydration or abnormality in electrolytes and skin lesions spread all over her body. It is generally known that inserting a catheter through exposed dermis increases the chance of bacterial infection. Accordingly, in such a case, it is better for doctors to avoid securing a central vein [11]. Moreover, doctors need to use an intraarterial catheterization to sample blood and to prevent skin damage caused by repetitive non-invasive measurement of blood pressure. Even in doing so, doctors should ensure that all catheters are inserted through normal areas of skin.

In our case, the patient showed stable vital signs and was not accompanied by fever or dehydration. However, she had severe skin lesions on both upper arms, so we decided to insert a catheter into the left radial artery, where skin regions are relatively small, in order to avoid repetitive non-invasive measurement of blood pressure. The possibility of regional anesthesia for TEN patients depends on the condition of the skin of anesthetic areas. However, there are some reported cases where people with SJS died of secondary infection of meningoencephalitis [6]. Therefore, when skin lesions are very severe, it is recommended to administer general anesthesia to prevent secondary infection. In our case, we also chose general anesthesia since she had skin lesions on her back, the region required to be anesthetized regionally. Anesthesiologists are recommended to use anesthetics that have minimal impact on the cardiovascular system, for instance etomidate and ketamine. However, we used propofol to our patient, considering that her hemodynamic condition was quite stable and applied mask on the face, with assisted ventilation, causiously to prevent skin peeling.

People with TEN are at risk of hyperkalemia [12], if they are given succinylcholine, a short-acting muscle relaxant. Nonetheless, we used the drug since the pregnant woman did not show any preoperative sign of fever or dehydration and abnormality in electrolytes, plus it was a Cesarean section. Aside from our case, however, we think that etomidate and rocuronium are safer if patients are judged to be susceptible to fever, dehydration, or abnormality in electrolytes by considering the results of blood and biochemistry tests, the degree of skin and mucous membrane infiltration, and clinical symptoms. We used isoflurane to maintain anesthesia in order to reduce liver damage, but meanwhile, there are some reported cases where total intravenous anesthesia with propofol and remifentanil is administered instead [11].

Furthermore, pneumothorax can occur due to intrapulmonary blebs, and the frequency of pneumothorax will increase as the positive pressure inside the thorax rises. Accordingly, we replaced $\mathrm{N}_{2} \mathrm{O}$ with air to minimize the airway edema and alveolar damage, and limited the tidal volume to $8 \mathrm{ml} / \mathrm{kg}$. We also conducted manual ventilation for the expulsion of the baby, in order not to raise airway pressure. Given that the depth of anesthesia is shallower when air is used instead of $\mathrm{N}_{2} \mathrm{O}$, we maintained the concentration of isoflurane at 1 MAC until the baby was fully delivered, in order to keep an appropriate depth of anesthesia. Extubation should be carefully carried out after considering how serious tracheal invasion is prior to a procedure and how serious pharyngolaryngeal edema is expected to be subsequent to a procedure. Doctors should be cautious not to damage the mucous membranes of an airway during extubation. Our patient had serious intraoral lesions and edema; however, she didn't get new scars during the endotracheal intubation and was categorized into Mallampati class 1 , meaning that her airway anatomy was favorable for an intubation. All in all, we decided to conduct an extubation while reviving her consciousness sufficiently. We chose not to conduct intraoral or endotracheal suction since they could give rise to fatal vesicles.

It is also important to control postoperative pain. To this end, we mixed Normal saline with 1,000 $\mu \mathrm{g}$ of fentanyl excluding ketorolac, which probably causes TEN, and supplied the mixture to the mother through an continuous infuser attached to her vein on the right leg. TEN patients are prone to hypothermia as their radiant heat is increased rapidly by the change in cutaneous blood flow and failure in controlling body temperature due to anesthesia. Accordingly, to prevent hypothermia, it is required to maintain the temperature of an operating room at $28^{\circ} \mathrm{C}$ and to provide a water mattress and forced air warmer along with warm fluid. In our case, we were unable to control the temperature of our operating room 
because our hospital building had central heating system. However, there was no problem since the operation duration was as short as 40 minutes and the room temperature was $24^{\circ} \mathrm{C}$, which is an acceptable level, and the patient's body temperature was stabilized.

Treatment for TEN consists of prompt diagnosis, discontinuation of suspected drug and appropriate symptomatic treatment and medication at specialized medical centers. Among drugs that were taken within 3 weeks after onset, those not critical for life support should be discontinued, while conducting conservative treatment that is equivalent to the one for burn patients. Some examples of conservative treatment are protection of the exposed dermis and erosed mucus membrane surfaces, early detection and treatment of the infection, an appropriate supply of fluid and prompt correction of electrolyte imbalances, nutrition supply and prevention of hypothermia, and proper pain control. Administration of preventive antibiotics has proved to be ineffective and it is typical to begin treatment after an infection is detected. Pulmonary complications frequently develop among TEN patients. If hypoxia is identified, doctors should suspect an invasion of respiratory epithelium and consider a supply of oxygen or endotracheal intubation, if necessary. For medication, administration of steroids or an immune supressant (e.g., cyclosporine), intravenous injection of immunoglobulin, and anti-TNFs are used. However, further studies are needed. Besides, insulin, zinc, and G-CSF are also well-known as treatment, and cases were reported that the disease was effectively healed through plasma exchange [13].

If TEN develops in a pregnant woman, it has no direct impact on her baby but causes premature labor pain [14]. As for the pregnant woman in this study, we stopped providing tocolytics, which was a suspected etiology for her TEN. After the discontinuation of the medication, the pain was not controlled but her lesions were rapidly aggravated, which, in turn, led to an emergency Caesarean section. Since serious complications were not found at that moment, there was little effect on the mother and the baby before and after the surgery. In conclusion, since TEN, though rare, is a highly progressive and fatal disease, it is necessary to check the level of severity of the invasion of skin, mucous membranes, and respiratory system, in order to choose a safe way of providing anesthesia in times of a Caesarean section. In particular, cautious care for the airway is needed for general anesthesia. Also, appropriate management should be taken (e.g., supply of fluid according to body conditions, correction of electrolytes, and prevention of hypothermia), thereby reducing postoperative morbidity and mortality.

\section{References}

1. Avakian R, Flowers FP, Araujo OE, Ramos-Caro FA. Toxic epidermal necrolysis: a review. J Am Acad Dermatol 1991; 25: 69-79.

2. Prins C, Kerdel FA, Padilla RS, Hunziker J, Chrimenti S, Viard I, et al. Treatment of toxic epidermal necrolysis with high-dose intravenous immunoglobulins: multicenter retrospective analysis of 48 consecutive cases. Arch Dermatol 2003; 139: 26-32.

3. Roujeau JC, Kelly JP, Naldi L, Rzany B, Stern RS, Anderson T, et al. Medication use and the risk of Stevens-Johnson syndrome or toxic epidermal necrolysis. N Engl J Med 1995; 333: 1600-7.

4. Choe YK, Nam HO, Lee JH, Lee KM, Cheong SH, Kim YJ, et al. Anesthetic management of a patient with toxic epidermal necrolysis: a case report. Korean J Anesthesiol 2005; 49: 433-7.

5. Dasgupta A, O'Malley J, Mallya R, Williams JG. Bronchial obstruction due to respiratory mucosal sloughing in toxic epidermal necrolysis. Thorax 1994; 49: 935-6.

6. Cucchiara RF, Dawson B. Anesthesia in Stevens-Johnson Syndrome: report of a case. Anesthesiology 1971; 35: 537-9.

7. Becker DS. Toxic epidermal necrolysis. Lancet 1998; 351: 1417-20.

8. Spies M, Sanford AP, Aili Low JF, Wolf SE, Herndon DN. Treatment of extensive toxic epidermal necrolysis in children. Pediatrics 2001; 108: 1162-8.

9. Claessens N, Delbeke L, Lambert J, Matthieu L, Lafaire C, Van Marck E. Toxic epidermal necrolysis associated with treatment for preterm labor. Dermatology 1998; 196: 461-2.

10. Paul C, Wolkenstein P, Adie H, Wechsler J, Garchon HJ, Revuz J, et al. Apoptosis as a mechanism of keratinocyte death in toxic epidermal necrolysis. Br J Dermatol 1996; 134: 710-4.

11. Şahin SH, Sevdi S, Güday I. Total intravenous anesthesia management of a patient with Stevens - Johnson Syndrome. J Turk Anaesth Int Care 2009; 37: 389-93.

12. Rabito SF, Sultana S, Konefal TS, Candido KD. Anesthetic management of toxic epidermal necrolysis: report of three adult cases. J Clin Anesth 2001; 13: 133-7.

13. Bamichas G, Natse T, Christidou F, Stangou M, Karagianni A, Koukourikos $\mathrm{S}$, et al. Plasma exchange in patients with toxic epidermal necrolysis. Ther Apher 2002; 6: 225-8.

14. Pacheco H, Araujo T, Kerdel F. Toxic epidermal necrolysis in a pregnant, HIV-infected woman. Int J Dermatol 2002; 41: 600-1. 